\title{
What impact do healthcare digital libraries have? An evaluation of national resource of infection control at the point of care using the Impact-ED framework
}

\author{
Patty Kostkova • Gemma Madle
}

\begin{abstract}
Over the last decade billions of dollars' worth of investments have been directed into ICT solutions for healthcare. In particular, new evidence-based digital libraries and web portals designed to keep busy clinicians up to date with the latest evidence were created in the UK and US. While usability and performance of digital libraries were widely researched, evaluation of impact did not seem to be sufficiently addressed. This is of major concern for healthcare digital libraries as their success or failure has a direct impact on patients' health, clinical practice, government policies and funding initiatives. In order to fill this gap, we developed the Impact-ED evaluation framework measuring impact on four dimensions of digital libraries - content, community, services and technology. Applying a triangulation technique we analysed pre- and post-visit questionnaires to assess the clinical query or aim of the visit and subsequent satisfaction with each visit, mapped it against weblogs analysis for each session and triangulated with data from semi-structured interviews. In this paper, we present the complete description of the Impact-ED framework, a definition of the comparative Impact score and application of the framework to a real-world medical digital library, the National Resource of Infection Control (NRIC, http:// www.nric.org.uk), to evaluate its impact at the point of care and demonstrate the generalisability of this novel methodology. We analysed the data from a cohort of 53 users who completed the registration questionnaire, of which 32 completed pre- and post-visit questionnaires of which 72 sets were matched for analysis and five users out of these
\end{abstract}

P. Kostkova (B)' G. Madle

City eHealth Research Centre, City University,

Northampton Square, EC1V OHB London, UK

e-mail: patty@soi.city.ac.uk were interviewed using Dervin's method. NRIC is generally perceived to be a useful resource with $93 \%$ of users reporting it provides relevant information regularly or occasionally $(n=28)$ and provided relevant information in over $65 \%$ of visits $(n=47)$. NRIC has a positive impact on user knowledge in over half of visits to the library $(52.8 \%)$, NRIC actual impact score $I_{\mathrm{a}}=0.65$ and the study revealed several areas for potential development to increase its impact.

Keywords Digital library Weblogs analysis National Resource of Infection Control Digital library evaluation Impact on clinical practice Impact evaluation Triangulation

$\begin{array}{ll}\text { Abbreviations } & \\ \text { NRIC } & \text { National Resource for Infection Control } \\ \text { DH } & \text { Department of Health, UK } \\ \text { NAO } & \text { National Audit Office, UK } \\ \text { NeLI } & \text { National electronic Library of Infection } \\ \text { DL } & \text { Digital library } \\ \text { IPS } & \text { Infection Prevention Society } \\ \text { DC } & \text { Dublin Core } \\ \text { IC } & \text { Infection control } \\ \text { UK } & \text { United Kingdom } \\ \text { NRIC AB } & \text { NRIC Advisory Board } \\ \text { RA } & \text { Reviewers' Assessment }\end{array}$

\section{Introduction}

Online digital library development is a relatively new area of research, and previous focus has been mostly on 
developmental issues or usability [1,3]. Digital libraries themselves are not consistently defined entities [2] and therefore evaluation has taken a back seat whilst developmental research has driven the field. But the field is now much more established with the term "digital library" widely used, with a simple Google search for this phrase returning 106 million results. More specifically a basic keyword search for "digital library" in the ACM Digital Library shows the growth in digital library publications over time as follows:

- Between 1952 and 2000 only 4,037 publications were published

- Between 2001 and 2004 there were an additional 6,979 publications

- But between 2005 and 2008 the figure was 14,130.

With more research being undertaken, the focus in digital library evaluation has started to shift towards impact evaluation [3] and this development has been mirrored in traditional library [4] domains.

But what is meant by impact and how can it be measured? A recent review of healthcare digital library impact evaluations identified a lack of a robust methodology, with most studies relying on self-reported user satisfaction scales as impact measurements [17]. This paper aims to present a new model for digital library impact evaluation and a new combination of methods to collect appropriate data and demonstrate its applicability on an evaluation of a key infection control digital library developed in the UK, the National Resource for Infection Control (NRIC).

NRIC was launched in May 2005 in response to National Audit Office [5-7] recommendations for a national infection control manual or repository for national evidence-based guidelines and good practice examples. The project, funded by the Department of Health (UK) and endorsed by the UK National electronic Library of Infection (http://www.neli.

org.uk [8]), covers a broad range of infection prevention and control, and information on infectious diseases. NRIC is freely available at http://www.nric.org.uk [9] and an increasing number of users visit from 165 different countries. The importance of infection prevention, and control policies and guidance cannot be over-emphasised. They form the basis for all safe evidence-based practice in any healthcare setting, outline the reasons why certain practices are important, and also attempt to define all patient care within safe boundaries.

Over the 7 years since its launch in May 2005, NRIC has helped to disseminate the plethora of new Infection Prevention and Control guidance published, and has become a useful resource in both Primary and Secondary Care health care settings and social care as well as the independent sector in the UK and internationally. The traffic to NRIC has been steadily growing and international recognition increasing [10].
But what difference is NRIC making to those who use it? Previous research in the 1980s and early 1990s has shown that traditional hospital library services can contribute to patient care and clinical decision-making [11], [12]. However, these studies were often driven by the need for the library to assert its value in times of economic constraint, therefore the focus tended to be on efficiency of services, satisfaction with the library and its impact on decision-making through self-reporting by clinicians, rather than measuring any actual outcomes, e.g. knowledge and attitudes, length of hospital stay, prescribing rates or patient satisfaction. These are approaches that have been mirrored in evaluations since the advent of digital libraries [18]. By contrast, one study did focus on the impact of performing Medline searches on actual outcomes to show that searches performed earlier in the patient's hospital stay were associated with lower costs, charges and length of stay than those whose searches were performed later [13]. But these large-scale resource-intensive studies are not always within the scope of digital libraries budgets and staffing levels.

The need to evaluate the impact of NRIC on professionals in the infection prevention and control field is essential to measure its success and where future investment can be targeted to improve its impact on clinical practice. But a new approach was taken to develop an impact evaluation framework allowing impact evaluation not relying on simple user satisfaction ratings.

\section{Methods}

'Impact concerns long-term and sustainable changes introduced by a given intervention in the lives of beneficiaries. Impact can be related either to the specific objectives of an intervention or to unanticipated changes caused by an intervention; such unanticipated changes may also occur in the lives of people not belonging to the beneficiary group. Impact can be either positive or negative, the latter being equally important to be aware of' [14].

The problem with this measure of impact is that often no information is available about how the library is used, what changes occur as a result of use, or what services can be improved to improve impact. There is a need for a generalisable measure not detached from the real-world setting of the library users. Discussing our impact evaluation methodology is the aim of this section.

\subsection{Impact-ED evaluation framework}

When your users are geographically diverse, which is always the case for online portals, and anonymous it is impossible to see this impact first-hand. Digital library impact, according to our framework, is assessed by bringing together 
users' knowledge, attitude and planned behaviour. We do not intend to evaluate behaviour change. Our approach focuses on defining and assessing the impact of DL at the point of care but measuring a subsequent users' behaviour change is beyond the scope of our IT-focused research. This would have required an independent evaluation data collection, typically, a resource demanding human observation, and control groups stipulating and assessing other behaviour influencing factors.

\subsection{Knowledge and attitude}

Understanding and defining knowledge is no easy task. Our aim is purely to present a summary of how current thinking about knowledge can relate to digital library impact evaluation, not to present a theory of knowledge. In our research the key question to be answered about knowledge is whether digital library users can use the library to gain knowledge from the information stored in the library documents. Regardless of which approach to describing the relationships between knowledge and information you prefer, a library needs to enable its users to access either data or information to result in a gain in knowledge. This sharing of what is termed "explicit knowledge" (i.e. knowledge that can be written down) [15] could be considered a fundamental aim of digital libraries, in order to equip users with the knowledge necessary to carry out their work, whether that be appropriate clinical guidelines, relevant articles for an assignment or evidence to support decisionmaking.

Attitude research has also attracted significant attention from the academic world with key psychologists Fishbein and Azjen suggesting that: "Attitude refers to a person's favourable or unfavourable evaluation of an object, event or person" [22].

Later in his Theory of Planned Behaviour, Azjen defines attitude toward a behaviour as:

"...the degree to which performance of the behavior is positively or negatively valued" [16].

There is general consensus on the definition of attitude, that it involves placing value or judgement on something or someone. In a digital library context attitudes are important as the value or judgement a healthcare professional places on the information held within the library may affect the impact this information has on their work [22].

However, measuring knowledge and attitude changes of digital library online users should not be performed by asking them to recall if the library has ever had an impact on their knowledge or their work, as has been the case for most previous digital library impact evaluations [17], but by actually measuring their specific knowledge and attitude changes at the point of use in real-time, using pre- and post-questionnaires. This approach has been piloted with a small digital library in the healthcare domain where library users were asked a series of questions before using the library and then asked the same questions after using the library, showing positive changes in knowledge and attitude [18,19].

Various research findings in psychology suggest that knowledge and attitude can be indicators of behaviour [20,21], and therefore this research uses them as proxy measures for digital library impact. Traditional methods of measuring knowledge and attitudes are scale measurements such as the Likert scale [22]. However, a new approach was required to provide more information about the impact of digital libraries in the healthcare domain.

\subsection{The theory of planned behaviour}

Dervin's model is, as Wilson describes "a model of methodology, rather than a model of a set of activities or a situation" [23]. Dervin's sense-making model [24] is considered to be a model of the "how to" of information seeking, unlike other information seeking models. It provides a step-by-step approach to evaluating how users bridge their information gap. Using the sense-making approach to inform questionnaire design in digital library impact evaluation will result in questionnaires that investigate how the user tries to build a bridge over their information gap by using the digital library and how this applies to their situation rather than just asking them factual or Likert scale questions.

While others investigated the broader effects of technology on medicine from sociological, economical and policy making perspectives [25], our focus remains on the user. The holistic approach we are proposing in this research investigates the knowledge provided by the DL in the decision making context of the individual user and directly at workplace.

\subsection{The Impact-ED model}

The Impact-ED (Impact E valuation for Digital Libraries) model on which the methods for evaluation were developed is shown in Fig. 1. It was developed to meet a set of impact evaluation criteria developed in a systematic review of digital library evaluations [17] and the full details can be found in [17,27].

The model is based around previously published digital library dimensions [26] but the model itself and its interactions is a new development as part of this research [27]. The intention of the Impact-ED model is that a variety of methods are used to collect data and data is linked to provide a more rounded picture of a digital library impact. 


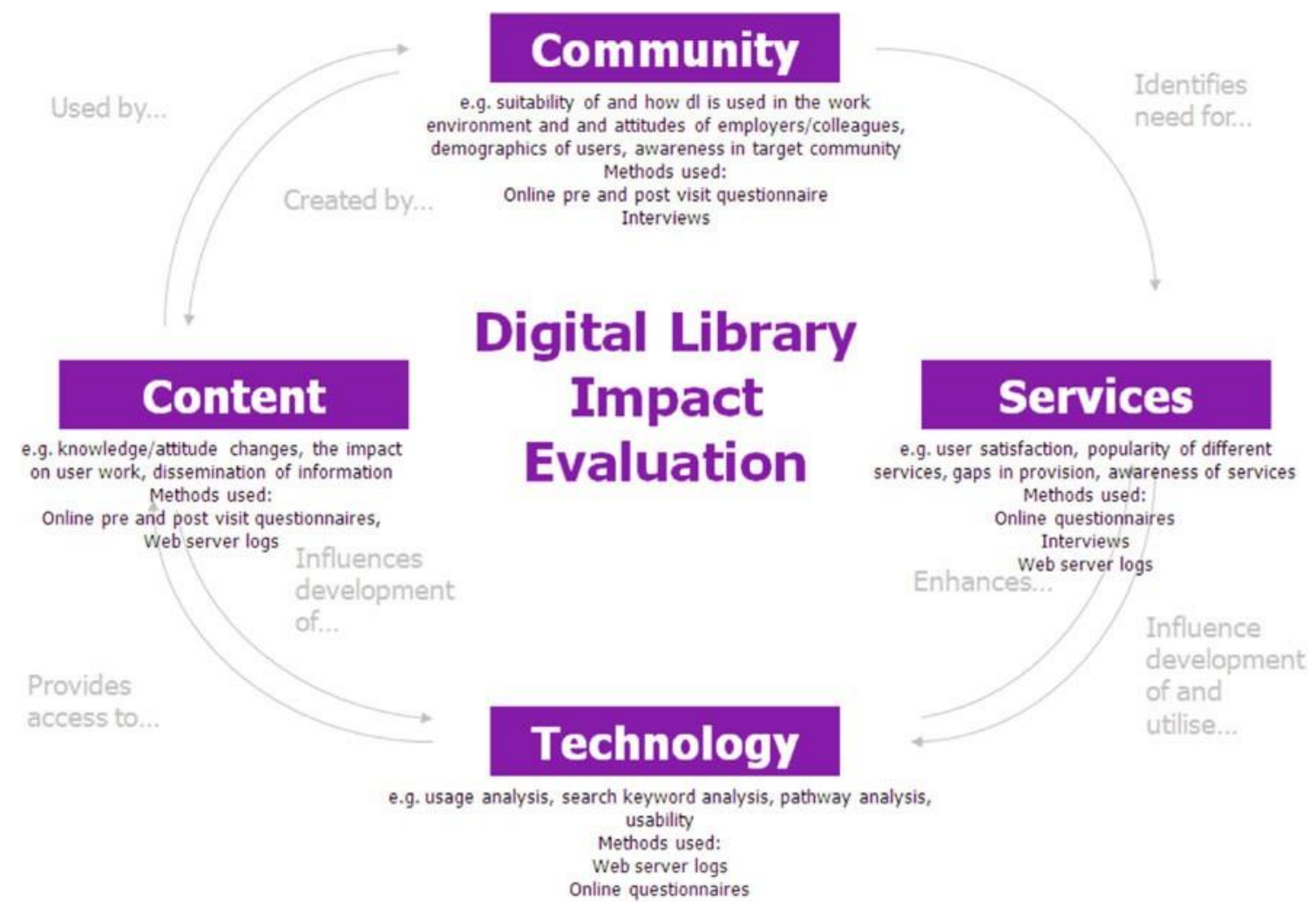

Fig. 1 Impact-ED impact evaluation framework

This will enable development of an impact score if evaluations are consistently based around one model [28].

Impact-ED provides a set of criteria around which questionnaires and interviews are designed to collect appropriate data. The criteria were developed by reviewing previous work in digital library evaluation and collating evaluation criteria that had been used. They are as follows:

\section{Community:}

1.1. How the digital library is used in the work setting and what are the reasons for use

1.2. The suitability of the work environment for integrating the digital library into work practice e.g. access to the Internet

1.3. Acceptability of the digital library by employers/ colleagues i.e. support given for using the digital library in work time and space

1.4. Awareness of the digital library in the target community, not just those who use it already

1.5. Basic demographic information of users

\section{Services:}

2.1. User satisfaction with the digital library and how it meets user needs
2.2. Preferred resources/services already offered by the digital library

2.3. Awareness of current digital library services and content to existing users

2.4. Gaps in provision

3. Technology:

3.1. Basic web access log statistics

3.2. Usability of the digital library

3.3. Search query analysis i.e. how are people searching the digital library, for what topics/parameters and are they finding what they are looking for

3.4. Navigation pathway analysis to identify how users are navigating the digital library and what services are commonly used

4. Content:

4.1. Knowledge and attitude changes as a result of using the digital library e.g. actual or self-reported

4.2. The impact of using the digital library on user work e.g. clinical decision-making and patient care, use in creating documents such as policies, guidelines or coursework

4.3. The dissemination of information found in the digital library to others 


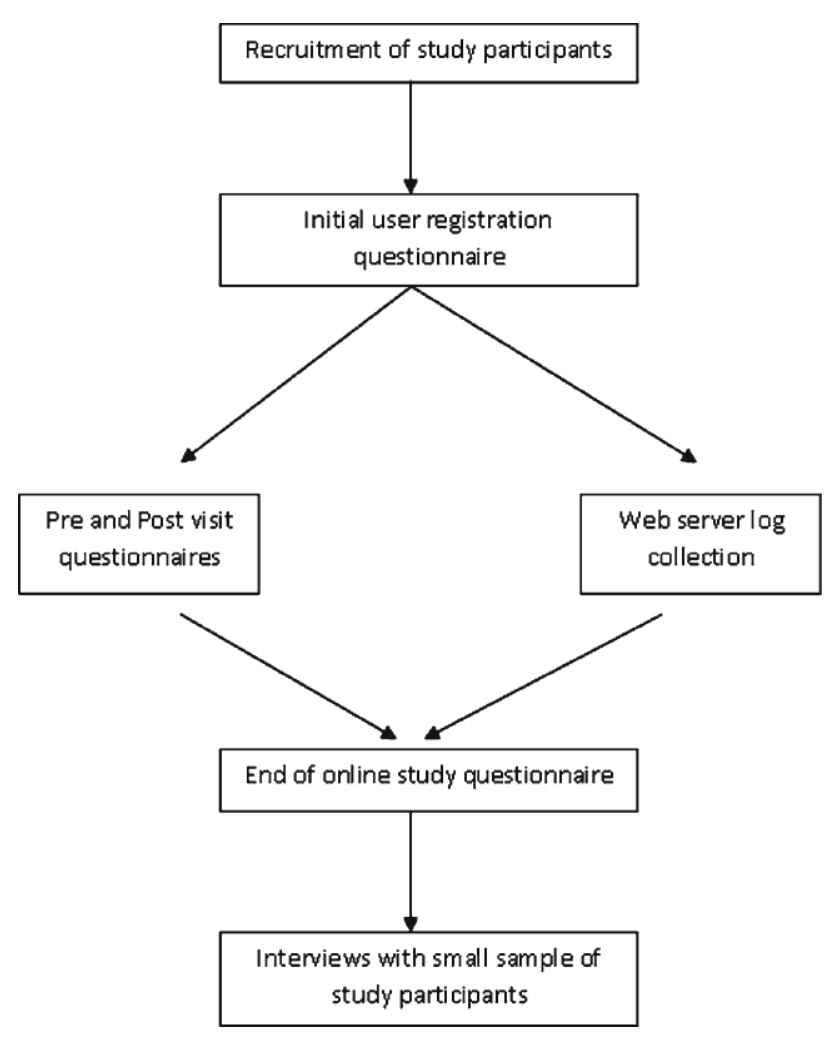

Fig. 2 Framework for digital library impact evaluation methods

2.5 Data collection methods

The methods used in the model are as follows:

1. Online questionnaires - investigating use of the DL within the work environment

2. Online pre- and post-visit (sense-making) questionnaires -investigating real-time, real-world use and how knowledge and attitudes change

3. Online tasks - how users complete tasks to find information within the library and how this changes knowledge and attitudes

4. Weblog analysis - shows what users actually did within the DL

5. Interviews - to compliment these other methods by providing more in-depth qualitative data that expands on issues identified in the questionnaires and weblogs.

Figure 2 illustrates how the DL evaluation methods are used together in a study flow diagram.

\section{Data analysis and the impact score}

The data set collected from the questionnaires, weblogs and interviews are triangulated using the following methods for each criterion in the four dimensions of the DL, as defined in the previous section. Weblogs were statistically analysed to calculate length and time of visits in terms of numbers, percentages and means. Statistical tests such as Fishers exact, independent $t$ test, ANOVA were used to evaluate the pre- and post-questionnaire data and qualitative analysis was applied on interview results to determine the outcome for each criterion from all three data sets.

Finally, an impact score is defined and calculated. Data was obtained from the pre- and post-visit questionnaires and coded to show where there was a strengthening of knowledge or change in knowledge or gain in knowledge as a result of a visit to the library. For each visit where this occurred the library scored 1 . A running total was kept until all visits had been scored and this was then divided by the total number of visits analysed. The calculation is shown below:

$V_{\mathrm{t}}=$ total number of visits analysed

$K=$ knowledge score (where $K=$ sum of number of visits where either a change/strengthening or gain in knowledge is recorded)

Impactscore $I=K / V_{\mathrm{t}}$

A key feature of the Impact-ED model and the digital library impact evaluation is the ability to see where the impact of the library can be improved as a part of formative evaluation. No impact evaluation can score an impact score of 1 as there will be reasons for the library having no impact on user knowledge during a visit over which it has no control e.g. user's poor internet connection causing time out, interruption to the users visit by something or someone external to the library. Therefore by recording reasons given by users where the library has no impact on user knowledge it is possible to predict a known maximum achievable score based on the areas in which the library has control i.e. if a reason for no impact is that the user could not find any information related to their query then the impact score could have been improved by either adding information where it was lacking or by improving the navigation or organisation of the library so the available information is more easily found. The calculation is as follows:

Reason for no impact $1\left(R_{1}\right)=$ e.g. No relevant information found

Reason for no impact $2\left(\mathrm{R}_{2}\right)=$ e.g. Could not access document

$\mathrm{R}_{3}$ etc

$V_{\mathrm{R}_{x}}=$ total number of visits with no impact coded $\mathrm{R}_{x}$

\section{Known maximum achievable impact score $I_{\max }=$} $\left(V_{\mathrm{R}_{x}}\right) / V+I$

Therefore the actual impact score $I_{r m ~ A}$ can be calculated as a ratio with the $I_{\max }$ as follows:

$$
I_{\mathrm{A}}=I / I_{\max }
$$

Using the definition, the $I_{\mathrm{A}}$ can also be calculated for all outputs or services rather than just an overall figure, for example: personal education, training/education of other staff etc. 


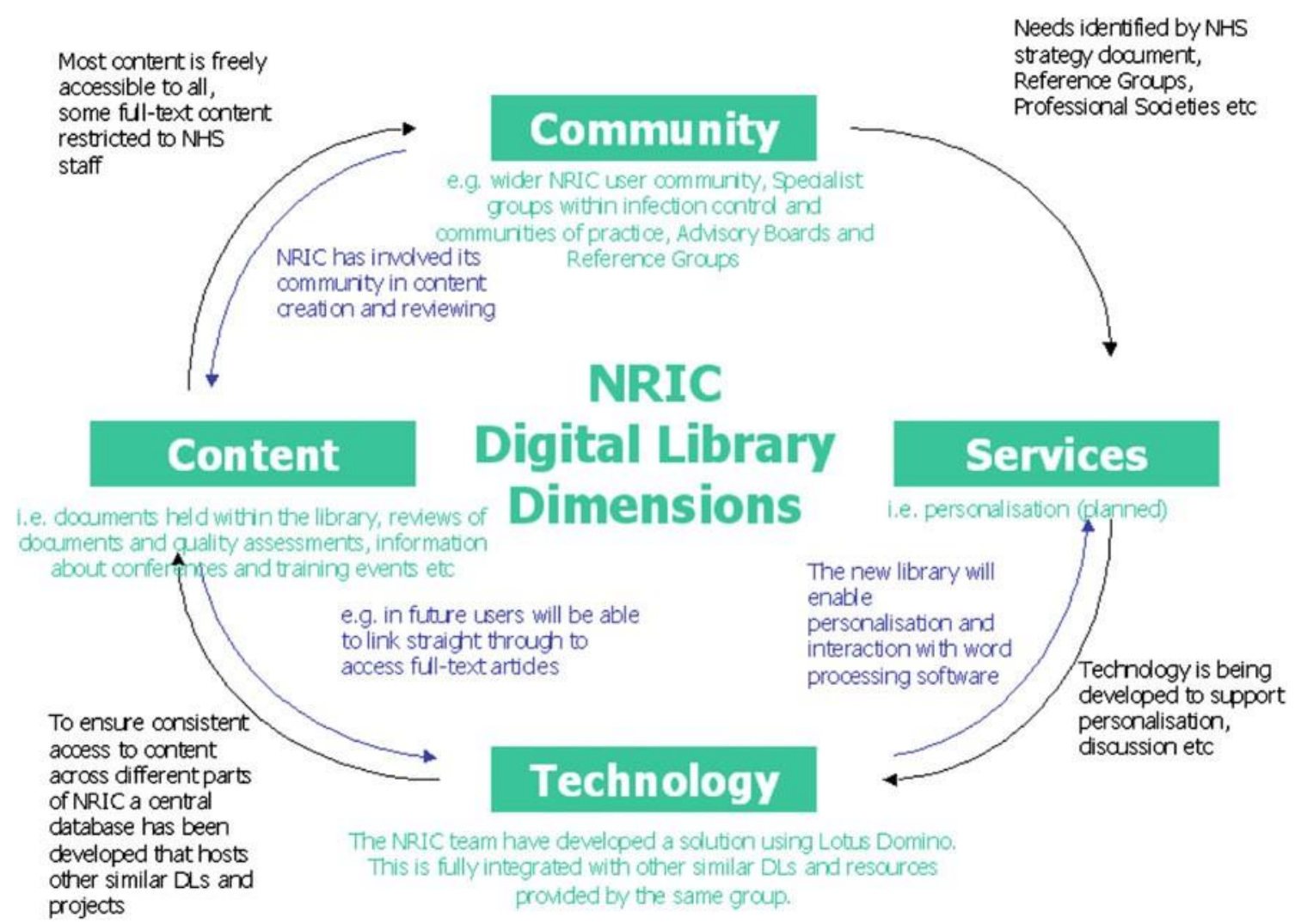

Fig. 3 Mapping the NRIC library onto the Impact-ED model

Therefore, as defined, Impact-ED provides a much more in-depth picture of how a digital library may be impacting its user community and their work than previous research has allowed.

\section{NRIC impact evaluation-Mapping Impact-ED model, data collection methods and user recruitment}

In order to use the Impact-ED model on NRIC, the framework needs to be mapped onto the specific situation of this digital library. Based on the generic model, Fig. 1, an NRICspecific mapping in accordance with the four dimensions was undertaken and can be found in Fig. 3.

The four dimensions of the Impact-ED model were applied on NRIC. The NRIC infection control (IC) community has been involved in identifying the need for services, and in some cases involved in creating and reviewing content for the library. The Internet technology, based on IBM Lotus Domino web server with a Dublin Core (DC) metadata tagged documents, was designed in order to provide the services required and to ensure consistent access to content throughout the library. The content is freely accessible to the community, although a minority of external documents have restricted access. The valued added feature of the NRIC service is the provision of evidence-based Review Assessment (RA) evaluating the soundness and evidence behind each resource and policy document. These are open for online discussions by registered members of the IC community. It is clear that there is a need for evaluation if the interactions between DL dimensions are to be effective. An obvious example is the influence of the content on the development of the technology. The content that will be or is being provided needs to be evaluated to allow improvement of the technology to increase the impact of the content on the user community.

The methods, adopted from the genetic Impact-ED model for this particular study, to collect data to meet the Impact-ED criteria were as follows:

- Study registration (Feb 2008) and end questionnaires (May 2008) - to find out who uses NRIC, how and when they were using it and for what reasons, compare answers before and after the study and to provide an opportunity for users to comment on services and suggest improvements.

- Pre- and post-questionnaire (Feb-May 2008) - to discover for what reasons people use NRIC at the point of use in their own words, what they know already to compare with what they think they have learnt from using NRIC and how they will apply this to their work

- Web server log collection (Feb-May 2008) - to collect data on how the participants actually navigate the library and see how this compares with how they report using it and the impact it has on their work Interviews 
(July-Aug 2008) - to provide more in-depth information in user's own words about how the site has an impact and how it can be improved

The methods were designed to collect data to meet all the evaluation criteria of the framework and Sect. 4 presents the results for each criteria.

Finally, in order to evaluate NRIC in clinical practice, potential participants from the target user group had to be recruited. Methods for recruitment included an advert on the NRIC website, the NRIC mailing list (approximately 1,800 subscribers in 2008), a post on the Infection Prevention Society (IPS) website forums and through contacts in the IPS South West branch.

\section{Results}

\subsection{Study setup}

As indicated in the previous section, the timeframe for the study was as follows: the study ran from February 2008 to May 2008 with interviews taking place during July and August 2008. The results were evaluated at the end of 2008 and presented to the project funder, DH, UK. Further, the study results and recommendations were officially discussed at the NRIC Annual Advisory Board meeting in 2009 and improvements to increase the impact of the library were implemented throughout the year 2010.

Sixty-five NRIC users signed up for the impact evaluation. Of these, two officially dropped out, 53 completed the registration questionnaire, 32 completed pre- and post-visit questionnaires of which 72 sets were matched for analysis, and 31 completed the end of study questionnaire. In addition, 5 out of the 53 users were interviewed using Dervin's method.

On arriving at the NRIC library, participants were asked to login with a unique username and password, purely for the purposes of tracking their activity during the study period and linking data from different collection methods.

Some data were subsequently statistically analysed to produce the so-called Impact Factor [17], however, the rich collection of qualitative and quantitative data, according to Impact-ED dimensions and methodology, was combined using the triangulations to provide a useful insight into the impact of NRIC on user work.

\subsection{IMPACT-ED evaluation according to the four dimensions}

In this section, we will discuss the impact of NRIC according to the criteria in the four main dimensions triangulating the

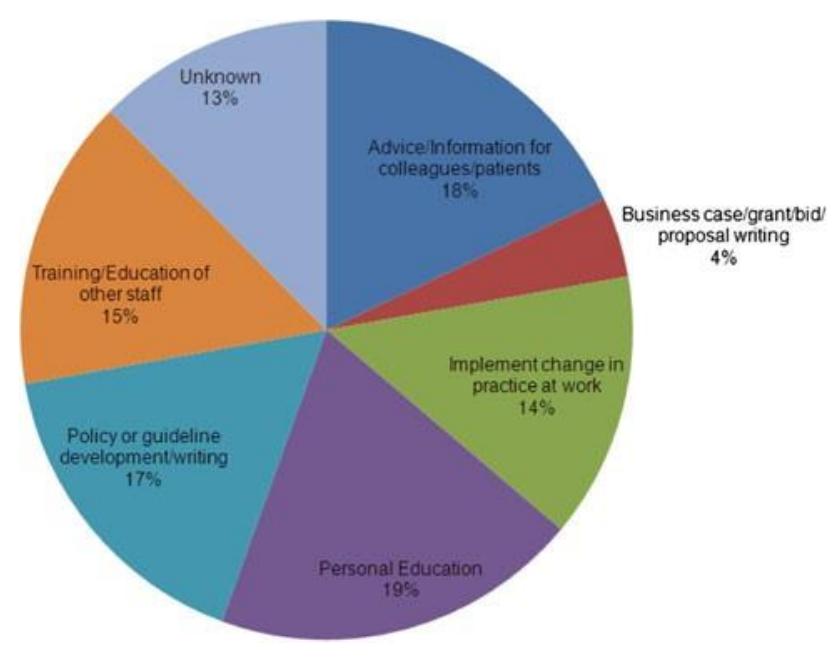

Fig. 4 Actual reasons for use for specific visits

three data collection methods: weblogs, questionnaires and interviews. We will focus on criteria of interest to the readership of the journal as some results are very infection control specific and therefore beyond the scope of this paper. The full details including all questionnaires used in this study can be found in [17].

\subsubsection{Community: how is NRIC used in the community?}

How the digital library is used in the work setting and what are the reasons for use NRIC is used mostly for finding evidence to support policy development, to implement a change in practice at work or for personal education (Fig. 4). In addition, a significant number of visitors use NRIC to search in the "what's new?" section and NRIC is often used in preference to source websites.

Combining the data sets, the interviews support the evidence from the questionnaires for the role of NRIC in policy development and personal education:

"As I said .....I also need evidence for the policies, ....And I find NRIC really useful for that because it's got the documents for everything in there. It's also got the policy templates and I find them very useful.”

\section{(Interviewee A)}

The suitability of the work environment for integrating the digital library into work practice e.g. access to the Internet \& Acceptability of the digital library by employers/colleagues i.e. support given for using the digital library in work time \& space For the reasons of simplicity for this paper, these two criteria were combined. Users generally felt that access to the Internet was easy in their workplace and there was a feeling of support from employers to use such resources although participants were more likely to agree with the former than 
Fig. 5 Reported use of four key services provided by the NRIC library (number of respondents is depicted on axe $x$ )

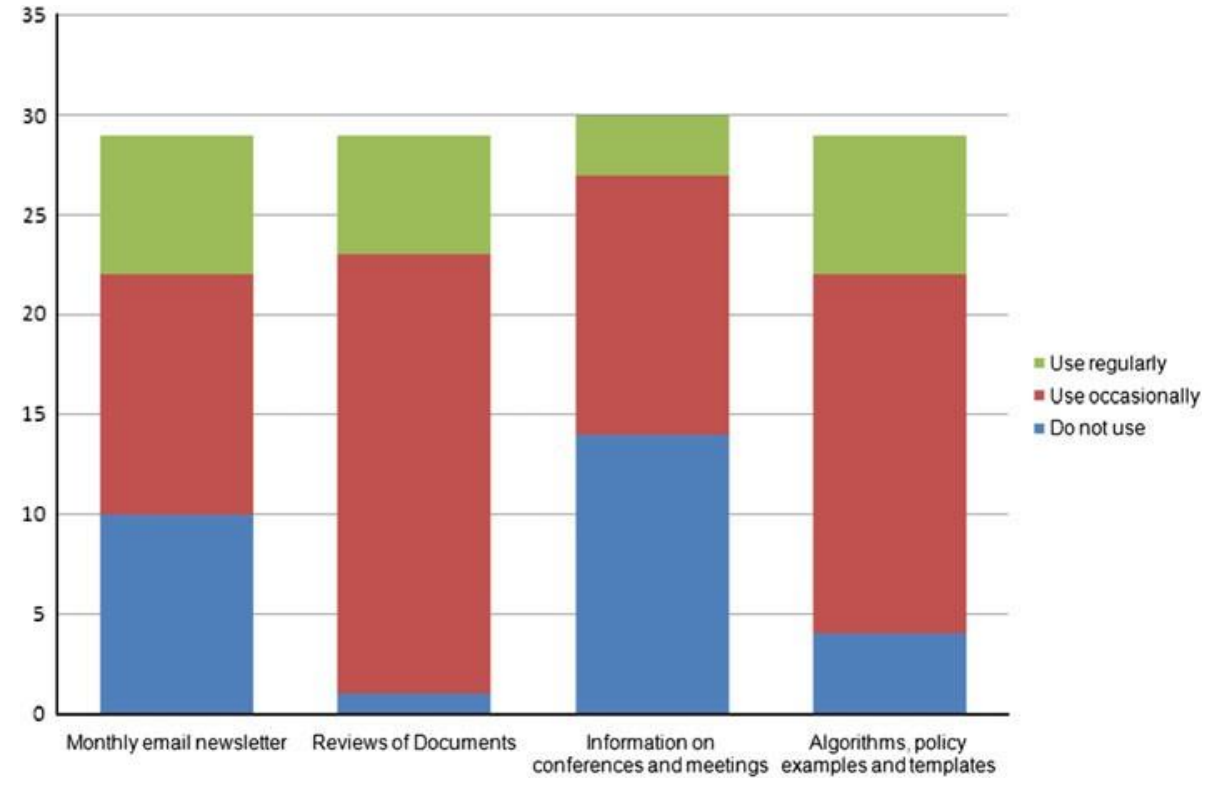

the latter, however, three interviewees reported distractions in the work environment.

Awareness of the digital library in the target community, not just those who use it already Most users disagreed or neither agreed nor disagreed with the statement that "Most of my colleagues are aware of NRIC and what it provides" with only $23 \%$ of those who answered (30) agreeing or strongly agreeing that this was the case.

Basic demographic information of users The majority of participants were nurses with 28 of the 52 responding that this was their profession. In reality this figure was probably higher as a number of participants entered "infection control specialist" or "infection control practitioner" and may well be nurses as well. Other professions included doctors, pharmacists, managers, and microbiologists. Thirty participants were definitely from the UK with 15 unspecified of which the majority were thought to be UK-based. Other countries represented included Spain, Saudi Arabia, the UAE and Egypt. The most common highest level of qualification was a taught postgraduate degree ( $n=1835 \%$ ), which is not an uncommon educational degree for senior nurses in the UK, and $63 \%$ ( $n$ 3) stated number of years of experience in their profession was over 10 years suggesting the study participants were a group of higher level staff who are experienced in their field.

\subsubsection{Services: how well received is NRIC and its services?}

User satisfaction with the digital library and how it meets user needs NRIC is generally perceived to be a useful resource with $93 \%$ of users reporting it provides relevant information either regularly or occasionally ( $\mp 28$ ) and provided relevant information in over $65 \%$ of visits ( 47 ). A regularly updated home page is important for attracting users. Clarification is required about the purpose of the document reviews (Reviewer's Assessments) as compared with the electronic catalogue card for each document.

"It makes a difference if I am trying to find out what is going on in the NHS"

(Interviewee D)

Preferred resources/services already offered by the digital library \& awareness of current digital library services and content to existing users Figure 5 shows the reported use of four key services provided by the NRIC library. The four services include: monthly email newsletter containing latest publications and upcoming conferences subscribed by over 3,500 users. In addition to providing evidencebased resource, NRIC provides a review of most documents (Review Assessment) in terms of evidence base practise. Information of upcoming conferences and meetings are available for the UK, Europe and key international events. NRIC also disseminates algorithms and national and local infection control policies, such as handwashing policy, decontamination policy compulsory in all UK Trusts. The Fig. 5 illustrates the frequency of use, however, awareness of all services but particularly the monthly newsletter and training courses/conferences information could be improved.

Gaps in provision Coverage of NRIC is generally perceived to be good with few gaps in content with $80 \%$ of respondents stating they felt there were few gaps in content $(n=$ 24). Popular suggestions for development include adding a 
Table 1 Basic access statistics

Basic access statistics for the 72 visits analysed

Mean time spent per visit

$00: 12: 22$

Mean number different pages viewed per visit

Mean number of documents viewed per visit

Median number of documents viewed per visit

3.07

Total number of reviews available

Number of reviews visited

$\%$ available reviews visited

$10.4 \%$

discussion board and developing the template policy section into national model policies.

\subsubsection{Technology: evaluation of usability and navigation behaviour}

Basic web access log statistics The users in this study spent a significant amount of time per visit (average over $12 \mathrm{~min}$ ) and visit on average three documents in one session, see Table 1. The independent $t$ test was performed to show that the more pages a user visited the more likely the library was to have an impact on their knowledge ( $F 0.002)$, time spent did not influence impact ( $p=0.118$ ) and the more documents a user viewed within the library the more likely NRIC was to have an impact $(p=0.006)$.

Usability of the digital library NRIC is generally perceived as an easy to use website but the main issue is with the display of the search results which at the time of the study were not ordered by date. It was suggested by some participants and interviewees that workshops could be run to increase awareness of NRIC and help users navigate it more effectively. But only $10.4 \%$ of available document reviews are accessed. The usability of the site, revealed at the interviews, was seems as very positive, for example:

"It is very easy to use and it's easy to get into".

(Interviewee A)

"It's a simple website...., it is a reliable site, you can get straight in there and go straight to mostly the documents you're looking for...

(Interviewee B)

Search query analysis i.e. how are people searching the digital library, for what topics/parameters and are they finding what they are looking for In the 44 visits that used the search facility 88 searches were performed. Only one of these searches used the filtering options provided on the search page to filter by publication type (a review). The most common way to search was to enter a phrase $(56.8 \%)$ or a single keyword (36.4\%) with only four search queries using a Boolean "AND" structure. Relevant information was found in $61.4 \%$ of visits that searched, however, in those that only searched and did not browse to navigate as well ( 717 ) information was found in only nine visits $(52.9 \%)$.

Navigation pathway analysis to identify how users are navigating the digital library and what services are commonly used In terms of getting around the library browsing is more common than searching and when NRIC is browsed rather than searched it is more likely to have an impact on user knowledge (Table 2).

Seventeen percent felt that the search results table could be improved, specifically to order by date of publication or allow sorting by categories of the user's choice, something supported by data from the interviews:

"the limitations are that the data capture can be far broader than the search terms, than the word terms typed in....: I think a main criticism is that, on the search facility (Interviewee B).

\subsubsection{Content: how is NRIC content perceived?}

Knowledge and attitude changes as a result of using the digital library e.g. actual or self-reported NRIC had an impact on user knowledge in $52.8 \%$ of visits - user knowledge was confirmed, strengthened or changed in $36.1 \%$ of visits and knowledge was gained by the user in $37.5 \%$ visits. In all there was an impact on user knowledge in $52.8 \%$ of the 72 visits. Where knowledge was gained most commonly this was adding to the user's existing knowledge about their query (20.8\% of visits), although on six occasions NRIC did add to user knowledge in unrelated areas. Where there was no impact on user knowledge reasons were investigated and are shown in Fig. 6.

The impact of using the digital library on user work e.g. clinical decision-making and patient care, use in creating documents such as polices, guidelines or coursework Following this case study evaluation an impact score calculation was developed and an impact score calculated for different areas of user work to show how NRIC is having an impact in these areas. Full details of the impact score of NRIC in the different work areas are discussed in the next section. As examples of NRIC having impact on responders work, we received mostly positive statements: "excellent for finding evidence to base new local policies on", "gives good up to date advice and resources" but also some negative views: "limited impact prefer using search engines on the internet which have a major impact on my clinical work".

"actually saved me a lot of time because the resources I need were all in one area. Instead of having to go into 
Table 2 Navigation strategy and impact (users may have confirmed knowledge and gained knowledge in the same visit therefore the total of users across each row may be greater than the number of users in each category)

\begin{tabular}{llll}
\hline $\begin{array}{l}\text { Category } \\
(n=\text { no. users) }\end{array}$ & $\begin{array}{l}\text { Confirmed/strengthened } \\
\text { or changed knowledge }(n)\end{array}$ & $\begin{array}{l}\text { Gained knowledge } \\
(n)\end{array}$ & $\begin{array}{l}\text { No impact } \\
(n)\end{array}$ \\
\hline Browsed only (24) & $45.8 \%(11)$ & $50.0 \%(12)$ & $37.5 \%(9)$ \\
Searched only (17) & $29.4 \%(5)$ & $23.5 \%(4)$ & $52.9 \%(9)$ \\
Browsed and searched (27) & $33.3 \%(9)$ & $37.0 \%(10)$ & $48.1 \%(13)$ \\
\hline
\end{tabular}

Fig. 6 Reasons for lack of impact of NRIC on user knowledge

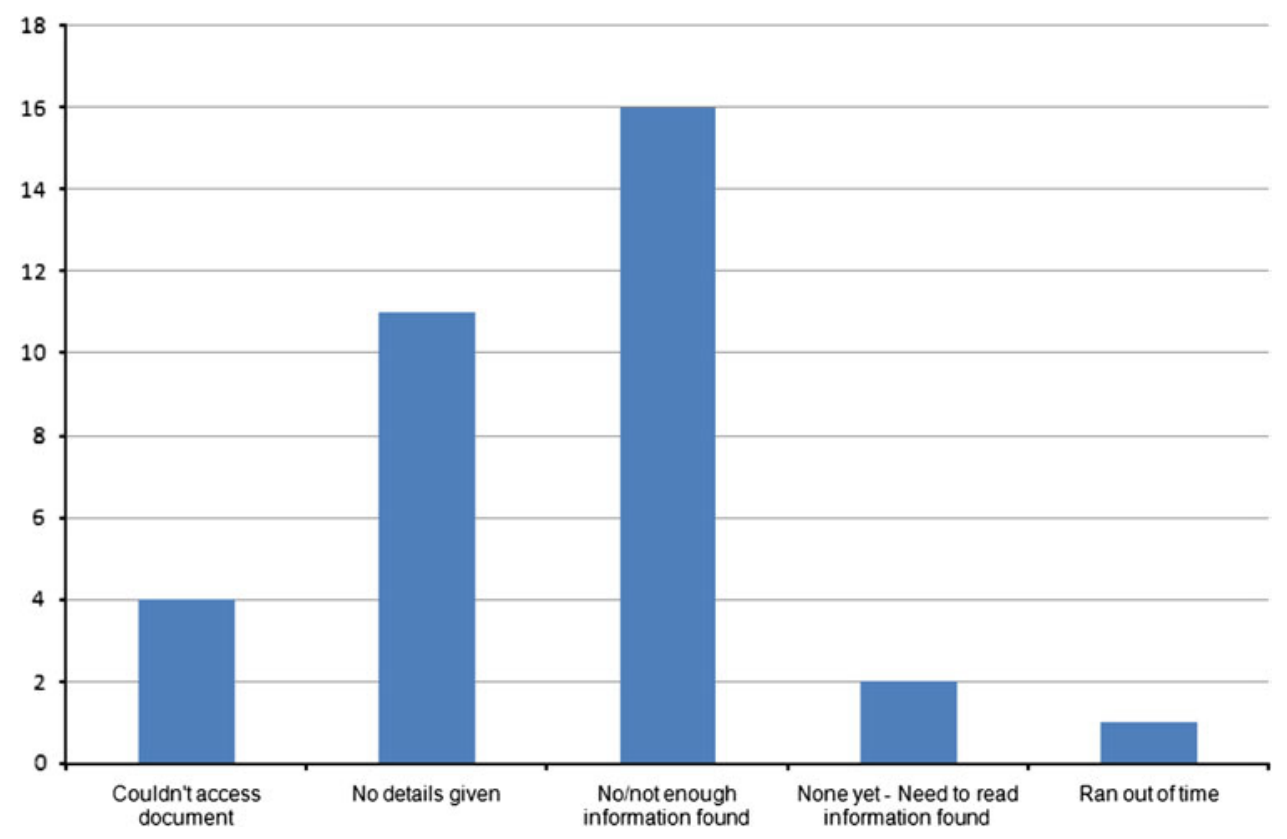

Google or whatever and look forever, or go across to the library....so that was good, saved me a lot of time".

(Interviewee A)

The dissemination of information found in the digital library to others Thirteen of the 72 visits were to find information to enable the user to pass on or give advice to other colleagues or patients. One specific visit was purely to find a url link to a Department of Health document for a colleague. In addition users direct others to the NRIC site rather than pass on the information themselves.

\subsection{Summary: NRIC's impact}

The very positive outcome of this study demonstrated that NRIC had an impact on user knowledge in $52.8 \%$ of visits ( $n$ 3). The main reasons for no impact were that not enough information was found $(n=16)$ or the user could not access the document $(n=4)$. NRIC has a positive impact in many areas of user work including policy development, training and education, implementing changes in practice and business case or proposal preparation.
Users also disseminated the NRIC evidence to other members of the community - therefore, knowledge having impact on the IC community. Thirteen of the 72 visits were to find information to enable the user to pass on or give advice to other colleagues or patients. One specific visit was purely to find a url link to a Department of Health document for a colleague. In addition users direct others to the NRIC site rather than pass on the information themselves.

...very often later on when I'm with staff ... they will tell me they've found something on NRIC even though I know I've told them about it but they've gone to NRIC and found it themselves and I think that probably makes it a bit more meaningful than me just trolling it out to them. So I think it is a useful resource for me to give to staff, clinical staff I think have used it quite a lot"

\section{(Interviewee D)}

\subsection{NRIC impact score}

As defined in the Impact-ED framework, there can be a maximum score of 1 where all visits would result in a change/strengthening or gain in knowledge. In the case of 
the NRIC library there were 38 visits where a strengthening, change or gain in knowledge was reported and a total of 72 visits therefore the first score was 0.53 .

$$
\begin{aligned}
& V_{\mathrm{t}}=72 \\
& K=38 \\
& I=38 / 72=0.53
\end{aligned}
$$

In the case of the NRIC library the reasons for no impact that could be influenced by the library were:

$R_{1}=$ no. of users who could not access document

$R_{2}=$ no. of users who reported no/not enough relevant information found

$$
\begin{aligned}
& V_{R_{1}}=4 \\
& V_{R_{2}}=16 \\
& I_{\max }=((4+16) / 72)+0.53=0.28+0.5=0.81
\end{aligned}
$$

Therefore the highest known achievable impact score for the NRIC library is 0.81 . Using this figure the $I_{\mathrm{A}}$ is:

$I_{\mathrm{A}}=0.53 / 0.81=0.65$

Figure 7 shows the impact of NRIC in different areas. In addition a significant proportion of NRIC use is to find information either on behalf of someone else or to pass information/advice on to patients or colleagues (IF 13) and interviewees also report directing others to NRIC to find information themselves.

In addition, Impact-ED evaluation should identify what library services and features influence impact and where a library could be improved the impact score calculation can be used to generate hypotheses that can then be validated by statistical tests. Data was collected for the following library features and services:

- Whether or not NRIC provided relevant information to the users query

- Whether or not users subscribed to the NRIC newsletter

- What navigation strategy users chose

- Whether users viewed the library's reviewer's assessments

Calculating impact scores for these different groups resulted in the hypotheses shown in Table 3. Statistical tests were performed to validate the data. The KolmogorovSmirnov test determined that the data was normally distributed.

Table 3 shows that if people find related information in NRIC then this does have an impact on their knowledge. However, there was no statistical significance for any of the other hypotheses despite the differences in impact scores. This is possibly due to the small sample numbers involved and a larger evaluation may provide more significant results. It does suggests that in the short term the single most important thing the NRIC library could do would be to improve its content coverage as there were a substantial number of visits (24 out of 72) where relevant information was not found by the user and should this be improved the impact score of NRIC would improve significantly.

\section{Discussion}

This research has shown that Impact-ED evaluation framework demonstrated that NRIC does have a positive impact on users knowledge and is contributing, according to all four dimensions of DL service, to the infection prevention and control community.

Several recommendations were made to the NRIC Advisory Board as a result of this study and were valid for improvements to NRIC as well as provide a rich recommendation for any Internet-based medical resource and portal. Recommended changes were implemented and NRIC continues providing the best available evidence to infection control professionals around the world: NRIC delivered information to over a quarter of a million users from 159 different countries in 2010 alone, is receiving over 20,000 hits per month, had positive impact on $53 \%$ visits, ranks in Top 10 in Google searches for infection-related terms and its subscription base has grown to over 3,500. In 2012, NRIC is fully established as an essential resource for busy infection control nurses around the world who are empowered by the latest evidence at their fingerprints helping them to keep patients safe from infections. Also, a new social media were recently implemented to further outreach to user base (NRIC has a twitter account and a page on Facebook [29] with 152 Fans in June 2012).

\subsection{Comparison to previous work}

Compared to previous work this evaluation takes a new approach. Previous healthcare digital library evaluation studies have measured impact in terms of self-reported changes in decision-making both retrospectively and prospectively either by multiple choice questions with or without comments or by interviews [17]. The Online Electronic Help evaluation [30] measured impact in terms of influence of the digital library on clinical decision-making in a scenario presented to the participant. The NASA Astronomy Data Services Digital Library evaluation [31] measured impact in terms of utility time i.e. time saved. Whilst retrospectively self-reported changes in decision-making and the impact of a digital library on decision-making in a scenario are both valid methods of impact measurement, neither are sufficient alone to determine the impact of a digital library on its users. The former is relying on users either remembering whether the digital library influenced their decision or reporting their expectations for the impact of the digital library in the future. Both of these may result in a more positive rather than accurate result as users overestimate the future impact of the digital library or report on a single incident (not necessarily a typical incident) of digital library use where the digital library had an impact on their decision-making. The measure of changes in decision-making with use of a digital library using a clinical scenario is an indicator of whether the digital library has the 


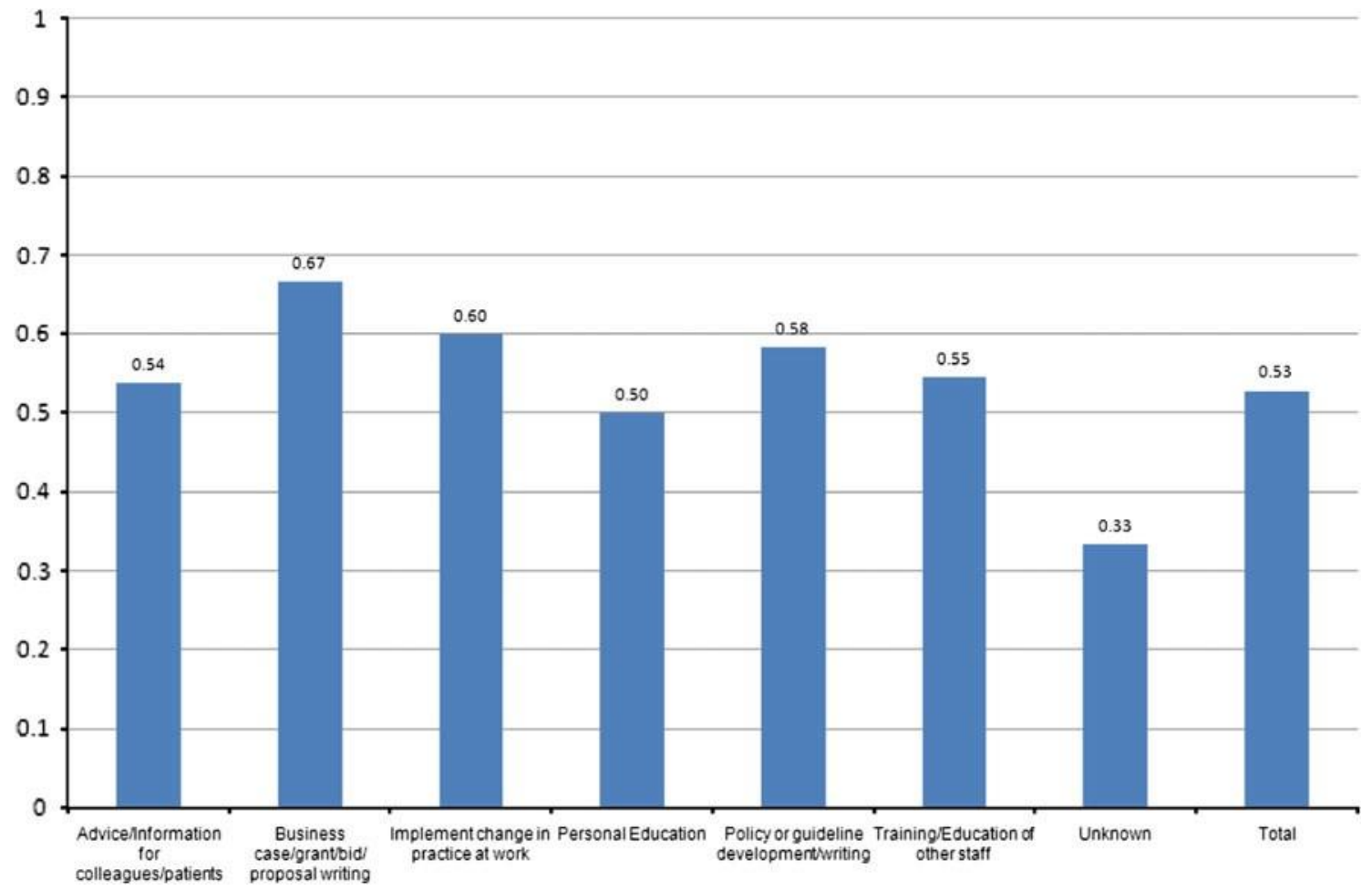

Fig. 7 Impact of NRIC in different work areas (a maximum score of 1 is possible for each area)

Table 3 Statistical significance of the impact of NRIC services and features

\begin{tabular}{|c|c|c|c|}
\hline Hypothesis & Impact scores $(I)$ & Test & $P$ value \\
\hline $\begin{array}{l}\text { When information is found in NRIC it has } \\
\text { an impact on user knowledge }\end{array}$ & $\begin{array}{l}\text { For visits when information was found } \\
\quad(n=47)=0.74 \\
\text { For visits when information was not found } \\
\quad(n=24)=0.13\end{array}$ & Fishers exact & $<0.0001$ \\
\hline $\begin{array}{l}\text { NRIC has a greater impact on its } \\
\text { newsletter readers than on } \\
\text { non-subscribers }\end{array}$ & $\begin{array}{l}\text { For visits by newsletter subscribers } \\
\quad(n=24)=0.55 \text { For visits by } \\
\text { non-subscribers }(n=10)=0.48\end{array}$ & Independent $t$ test & $<0.5$ \\
\hline $\begin{array}{l}\text { NRIC has a greater impact on visitors who } \\
\text { browse rather than search or do both }\end{array}$ & $\begin{array}{l}\text { Browsing only }(n=24)=0.63 \text { Search } \\
\text { only }(n=17)=0.47 \text { Browse and } \\
\text { search }(n=27)=0.52\end{array}$ & ANOVA & $<0.5$ \\
\hline $\begin{array}{l}\text { NRIC has a greater impact on visitors } \\
\text { who view reviewer's assessments than } \\
\text { those who don't }\end{array}$ & $\begin{array}{l}\text { View reviewer's assessments } \\
(n=5)=0.6 \text { Didn'tview } \\
\text { reviewer's assessments } \\
(n=29)=0.52\end{array}$ & Independent $t$ test & $<0.5$ \\
\hline
\end{tabular}

potential to aid decision-making in clinical practice but cannot be wholly representative of this as it is not subject to the constraints that may be present in the environment in which the user may be accessing the digital library from in the realworld e.g. busy hospital ward or GP surgery. Therefore the approach taken by this research to evaluate knowledge and attitude changes at the point of use, gives a more representative indication of the impact of the NRIC library on user work than previous work would have allowed.

\subsection{Limitations of this approach}

Users who took part of this study were volunteers by our recruitment channels. As a substantial part of the Impact-ED evaluation is qualitative and to certain level personalised, the framework does not intend to statistically analyse the results to all NRIC users, however, a larger and more geographically and profession-wise diverse sample would provide richer data. Expanding the study inthis way would be too costly and 
therefore unfeasible as a research project, unless specifically externally funded. Further, as the users were self-selected it is likely that these volunteers were more enthusiastic in using technology in their clinical practise and therefore present a certain bias to the study.

As we relied on self-reported attitudes and behaviour, there is a known issue with a discrepancy between user selfperceived behaviour and real behaviour [32,33], however, without expensive resource demanding observational studies self-reporting is the only way to collect data from user.

There are problems in assuming that a change in attitude always leads to a change in behaviour. However, with the limitations of the project in terms of following up users to record actual behaviour and with the evaluation investigating specific queries from users in real world environments it is the best fit proxy measure available within the constraints of the project, building on work by Dervin at al. [24]. The model allows for data collection about specific intended behaviour as a result of a specific attitude change, a factor that is suggested to increase the likelihood of the behaviour being carried out. A full discussion of the validity of this approach to impact evaluation is published in [17].

When evaluating libraries such as the NRIC library where the library points to external content rather than providing content itself, it is more difficult to track user activity. However, by asking the user in the post visit questionnaires which documents or resources they used and linking this to catalogue cards viewed we can see which library resources are being used and their relation to impact. There is the potential for users to navigate outside the library once on an external resource and find information elsewhere but in order to complete the questionnaire they always return. Also if their journey to the external information began inside the library the argument could be that the library has influenced their knowledge and attitudes by leading them to a resource which led them potentially elsewhere to their answer. Ideally we would track user activity on the whole World Wide Web during a session to investigate what users do when they leave the library but this is technically not possible as server logs are proprietary to organizations hosting the sites.

\section{Conclusion}

This paper discussed a novel evaluation framework, Impact$\mathrm{ED}$, assessing the impact of medical digital libraries in terms of impact on their community, technology, services and content. In this paper, we presented the framework and the evaluation criteria for the four dimensions and defined an impact score quantifying the impact of the DL. The results of an evaluation of the impact of a real world medical DL, the National Resources of Infection Control (NRIC) demonstrated how the Impact-ED methodology provides an in-depth assessment of a real-world medical portal. Using this new approach this paper has shown that NRIC is a popular, easy to use, library that is having a positive impact on $52.8 \%$ of user knowledge and work while the actual impact $I_{\mathrm{A}}$ of the NRIC library was 0.65 . However, further sustained investment is required if NRIC is to fulfil its potential as a one-stop resource in the infection prevention and control community in the UK and globally.

Acknowledgments Thanks to Gawesh Jawaheer for his contribution to the NRIC project and his help with running the evaluation on the NRIC website, Sue Wiseman and Abdul Roudsari for their involvement in the study. We also acknowledge David Fowler for proof-reading the manuscript. The work was supported by funding from the Department of Health UK for the NRIC project.

\section{References}

1. Chowdhury, S., Landoni, M., Gibb, F.: Usability and impact of digital libraries: a review. Online Inform. Rev. 30(6), 656-680 (2006)

2. Borgman, C.: Designing digital libraries for usability. In: Bishop, A. P., Van House, N. A. \& Buttenfield, B. P. (eds.) Digital library use: social practice and evaluation. MIT Press, Cambridge, pp. 85-118 (2003)

3. Chowdhury, G.G., Chowdhury, S.: Introduction to digital libraries. Facet Publishing, London (2003)

4. McNicol, S.: The eVALUEd toolkit: a framework for the qualitative evaluation of electronic information services. Vine 34(4), 172-175 (2004)

5. National Audit Office. Improving patient care by reducing the risk of hospital acquired infection: A progress report (A report by the Comptroller and Auditor General) (2004) HC 876. Available at: http://www.nao.org.uk/publications/nao_reports/03-04/ 0304876es.pdf (Summary) Archived at: http://www.webcitation. org/5wNaAjY2Q or http://www.nao.org.uk/publications/nao_ reports/03-04/0304876.pdf (Full Report) Archived at: http:// www.webcitation.org/5wNaXQTLW

6. National Audit Office. The Management and Control of Hospital Acquired Infection in Acute NHS Trusts in England. (2000) HC 230. Available at: http://www.nao.org.uk/publications/ nao_reports/9900230.pdf Archived at: http://www.webcitation. org/5wNadLzCS

7. National Audit Office. Reducing Healthcare Associated Infections in Hospitals in England (2009) HC 560. Available at: http:// www.nao.org.uk/idoc.ashx?docId=c1a1ff01-336f-494e-8c9623fa9bf8c0dc\&version=-1 Archived at: http://www.webcitation. org/5wNagVvOo

8. http://www.neli.org.uk Archived at: http://www.webcitation.org/ 5wNb7YbzN. Accessed 15 June 2012

9. http://www.nric.org.uk Archived at: http://www.webcitation.org/ 5wNbDCi7M. Accessed 15 June 2012

10. Wiseman S, Kostkova P, D'Souza S, Mani-Saada J, Madle G. Evidence-based infection control - a national resource, Br. J. Infect. Control. 17(3), 13-14 (2006)

11. Marshall, J.G.: The impact of the hospital library on clinical decision making: the Rochester study. Bull. Med. Libr. Assoc. 80, 169-178 (1992)

12. Connor, P.: Determining the impact of health library services on patient care: a review of the literature. Health Inf. Libr. J. 19, 1-13 (2002)

13. Klein, M.S., Ross, F.V., Adams, D.L., Gilbert, C.M.: Effect of online literature searching on length of stay and patient care costs. Acad. Med. 69, 489-495 (1994) 
14. Blankenberg, F.: Methods of impact assessment research programme: Resource pack and discussion paper for the case studies phase. Oxfam UK \& Novib, The Hague (1995)

15. Rowley, J.: The wisdom hierarchy: representations of the DIKW hierarchy. J. Inform. Sci. 33(2), 163-180 (2007)

16. Ajzen, I. The theory of planned behavior diagram. Icek Ajzen's Home Page. (2006). 11-12-2007. http://people.umass. edu/aizen/tpb.diag.html, Archived at: http://www.webcitation.org/ 68ZNljpcW. Accessed 20 June 2012

17. Madle, G.: Impact-ED: a new model of digital library evaluation. PhD Thesis, City University, London, (2009)

18. Madle, G., Kostkova. P., Mani-Saada, J., Weinberg, J. R.: Evaluating the changes in knowledge and attitudes of digital library users. Lecture Notes in Computer Science. Springer, Trondheim, pp. 29-40 (2003)

19. Madle, G., Kostkova, P., Mani-Saada, J., Weinberg, J., Williams, P.: Changing public attitudes to antibiotic prescribing: can the internet help? Informat. Prim. Care. 12(1), 19-26 (2004)

20. Ajzen, I., Manstead, A.S.R., et al.: Changing health-related behaviors: an approach based on the theory of planned behaviour. In: van den Bos, K. (ed.) The scope of social psychology: theory and applications, pp. 43-63. Psychology Press, New York (2007)

21. Ajzen, I.: The theory of planned behaviour. Org. Behav. Human Decis. Process. 50, 179-211 (1991)

22. Fishbein, M., Ajzen, I.: Belief, attitude, intention, and behavior: an introduction to theory and research. Addison-Wesley, Reading (1975)

23. Wilson, T.D.: Models in information behaviour research. J. Doc. 55(3), 249-270 (1999)

24. Dervin, B.: Audience as listener and learner, teacher and confidante: the sense-making approach. In: Dervin, B., ForemanWernet, L., Lauterbach, E. (eds.) Sense-making methodology reader: selected writings of Brenda Dervin, pp. 215-232. Hampton Press, Cresskill (2003)

25. Casper, M., Daniel, R., Morrison, D.R.: Medical sociology and technology: critical engagements. J. Health Soc. Behav. 51, 120132 (2010). doi:10.1177/0022146510383493
26. Fox, E., Marchionini, G.: Progress towards digital libraries: augmentation through integration. Inform. Process. Manag. 35, 219-225 (1999)

27. Madle, G., Kostkova, P., Roudsari. A.: Impact-ED — a new model of digital library impact evaluation. In: Proceedings of the ECDL 2008 conference, Aarhus, Denmark, September 2008. Lecture Notes in Computer Science, vol. 5173/2008, pp. 100-105. doi:10.1007/9783-540-87599-4

28. Kostkova, P., Madle, G.: User-centred evaluation model for medical digital libraries. In: Riaño, D. (ed.) K4HelP 2008. Lecture Notes in Artificial Intelligence, LNAI vol. 5626/2009, pp. 92-103 (2009). doi:10.1007/978-3-642-03262-2

29. http://www.facebook.com/pages/NRIC-National-Resource-forInfection-Control/322620358370?ref=mf Archived at: http:// www.webcitation.org/5wNanRZEc. Accessed 15 June 2012

30. Berkenstadt, H., Yusim, Y., Katznelson, R., Ziv, A., Livingstone, D., Perel, A.: A novel point-of-care information system reduces anaesthesiologists' errors while managing case scenarios. Eur. J. Anaesthesiol. 23(3), 239-250 (2006)

31. Accomazzi, A., Demleitner, M., Eichhorn, G., Grant, C., Kurtz, M.J., Murray, S.S.: Worldwide use and impact of the NASA astrophysics data system digital library. J. Am. Soc. Inform. Sci. Technol. 56(1), 36-45 (2005)

32. Roy, A., Kostkova, P., Catchpole, M., Carson, E.: Comparative study of user perceived and actual information searching behaviour in the National electronic library of infection. In: Kostkova, P. (ed.) ehealth 2009, Springer Lecture Notes of the Institute for Computer Sciences, Social-Informatics and Telecommunications Engineering LNICST 27, pp. 96-103 (2010)

33. Madle, G., Berger, A., Cognat, S., Menna, S., Kostkova, P.: 8. User information seeking behaviour: perceptions and reality. An evaluation of the WHO Labresources Internet portal. Inform. Health Soc. Care. 34(1), 30-38 (2009) 